\title{
Stress-Strain Relation in Shear From Twisting Test of Annulus ${ }^{1,2}$
}

\author{
Walter Ramberg and James A. Miller
}

\begin{abstract}
It is shown that the stress-strain relation in shear of isotropic thin sheet can be determined from a test of an annular specimen. The annulus must be clamped uniformly along the inner and the outer edge, and the relative twist of two circles concentric with the edges must be measured. The shear strain at the inner circle can be computed from the shear strain at the outer circle and the slope of the torque-twist curve. Tests were made on 0.032 inch aluminum alloy $75 \mathrm{~S}-\mathrm{T} 6$ sheet well beyond the elastic range to a shearing stress of 48,000 $\mathrm{lb} / \mathrm{in}^{2}$ EPON adhesive VI was used to bond the sheet to a clamping ring at the outer edge of the annulus and between the sheet and the end of a cylinder through which the torque was applied.
\end{abstract}

\section{Introduction}

The stress-strain relation in shear of thin sheet metal is of practical importance for estimating the strength of shear webs. It is of theoretical importance in studying the plastic behavior of metals under combined stress.

Many methods have been suggested for determining the stress-strain curve in shear of thin sheet [1], ${ }^{3}$ and at least two of them have been tried. Good results have been obtained so far only from twisting tests of square plates of aluminum alloy [1]. Unfortunately the twisting test of a square plate makes severe demands on the skill and patience of the test engineer. Furthermore, the specimen becomes too small for precision in the measurement of strain and in the application of the twisting loads for sheet less than $0.1 \mathrm{in}$. thick. Hence it seemed desirable to try out some of the other methods that have been proposed. One of the most promising among these is the twisting test of an annulus of constant thickness. This method was accordingly investigated at the National Bureau of Standards as part of a program supported by the Office of Naval Research, United States Department of the Navy.

\section{Theory of Twisting Test of Annulus of Constant Thickness}

Consider an annulus of constant thickness $h$, twisted by a torque $M_{t}$, which applies a constant shearing force per unit length along the inner edge $r=a$, figure 1 , and along the outer edge $r=b$. If the material is isotropic, the shearing stress at any radius $r$ is given by

$$
\tau=\frac{M_{t}}{2 \pi r^{2} h} .
$$

The shearing strain at any point can be derived from the radial displacement $u$ and the circumferential displacement $v$ by substitution in the equation

\footnotetext{
1 This paper is based on work sponsored by the Mechanics Branch, Office of Naval Research, U. S. Department of the Navy.

2 Presented at the Eighth International Congress on Theoretical and Applied Mechanics in Istanbul, Turkey, August 20-28, 1952.

${ }_{3}$ Figures in brackets indicate the literature references at the end of this paper.
}

for plane strain in polar coordinates $r, \theta[2$, p. 66]:

$$
\gamma=\frac{\partial v}{\partial r}+\frac{\partial u}{r \partial \theta}-\frac{v}{r}
$$

In an isotropic annulus the displacements $u, v$ caused by twisting are independent of $\theta$, so that (2) reduces to

$$
\gamma=\frac{d v}{d r}-\frac{v}{r}=r \frac{d}{d r}\left(\frac{v}{r}\right)
$$

Integration of this expression leads to a formula for the circumferential displacement $\delta$ of a point on an outer circle with radius $r_{2}$ relative to a platform resting on an inner circle with radius $r_{1}$, figure 1 . We note from figure 1 that this displacement is

$$
\delta=v_{2}-v_{1} \frac{r_{2}}{r_{1}}=r_{2}\left(\frac{v_{2}}{r_{2}}-\frac{v_{1}}{r_{1}}\right)
$$

Dividing $\gamma$ by $r$ in (3) and integrating gives

$$
\int_{r_{1}}^{r_{2}} \frac{\gamma}{r} d r=\frac{v_{2}}{r_{2}}-\frac{v_{1}}{r_{1}}
$$

so that

$$
\frac{\delta}{r_{2}}=\int_{r_{1}}^{r_{2}} \frac{\gamma}{r} d r
$$

From this an equation for the shearing strain $\gamma_{1}$ at the circle $r=r_{1}$ can be obtained as follows. We can replace $r$ as the independent variable in the integral by $\tau$, by taking the logarithms of the two sides in eq (1)

$$
\log \tau=\log \frac{M_{t}}{2 \pi h}-2 \log r
$$

and then differentiating for a given plate under given twisting moment $\left(M_{t}, h\right.$ constant):

$$
\frac{d \tau}{\tau}=-2 \frac{d r}{r}
$$




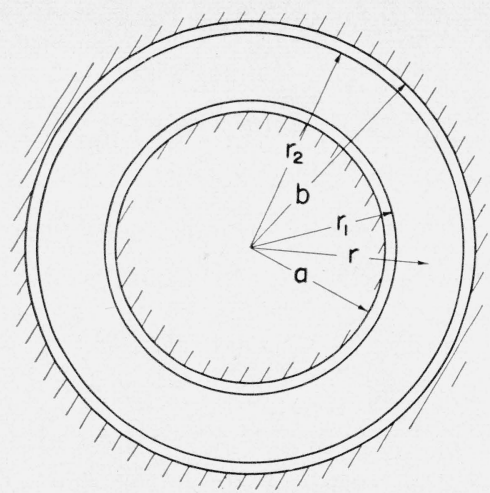

Figure 1. Diagrammatic sketch of annulus.

$a$, inner radius; $b$, outer radius; $r_{1}$, radius of inner gage circle; $r_{2}$, radius of outer gage circle.

Inserting (8) in (6) gives

$$
\delta=-\frac{r_{2}}{2} \int_{\tau_{1}}^{\tau_{2}} \frac{\gamma}{\tau} d \tau
$$

where $\tau_{1}, \tau_{2}$ are the shearing stresses at radii $r_{1}, r_{2}$, respectively. Differentiating with respect to $M_{t}$ gives [3, p. 29]

$$
\begin{array}{r}
\frac{d \delta}{d M_{t}}=-\frac{r_{2}}{2}\left[\int_{\tau_{1}}^{\tau_{2}} \frac{d}{d M_{t}}\left(\frac{\gamma}{\tau}\right) d \tau+\right. \\
\left.\frac{d \tau_{2}}{d M_{t}}\left(\frac{\gamma_{2}}{\tau_{2}}\right)-\frac{d \tau_{1}}{d M_{t}}\left(\frac{\gamma_{1}}{\tau_{1}}\right)\right] .
\end{array}
$$

Since the ratio of $\gamma$ to $\tau$ does not depend on the parameter $M_{t}, d(\gamma / \tau) / d M_{t}=0$, and consequently the integral on the right-hand side is equal to zero. The derivatives $d \tau_{2} / d M_{t}$ and $d \tau_{1} / d M_{t}$ are obtained directly from (1), noting that $r$ is equal to $r_{1}, r_{2}$, respectively. This leads to

$$
\frac{d \delta}{d M_{t}}=\frac{r_{2}}{4 \pi h}\left(\frac{\gamma_{1}}{\tau_{1} r_{1}^{2}}-\frac{\gamma_{2}}{\tau_{2} r_{2}^{2}}\right)
$$

This equation can be solved for the shearing strain $\gamma_{1}$ at the inner circle

$$
\gamma_{1}=\gamma_{2}+\frac{2}{r_{2}} M_{\imath} \frac{d \delta}{d M_{t}}
$$

Equation (12) makes possible a step-by-step construction of the stress-strain curve in shear, provided that the measurements are started within the elastic range. The shear modulus $G$ may be determined from a single measurement of $\delta$ in that range by noting that inside the elastic range

$$
\frac{\gamma_{2}}{\tau_{2}}=\frac{\gamma_{1}}{\tau_{1}}=\frac{1}{G}
$$

Inserting in (11) and solving for $G$ :

$$
G=\frac{r_{2}}{4 \pi h}\left(\frac{1}{r_{1}^{2}}-\frac{1}{r_{2}^{2}}\right) / \frac{d \delta}{d M_{t}} \text {. }
$$

\section{Procedure for Twisting Test}

\subsection{Dimensions of Specimen}

The dimensions of the specimen must be chosen to satisfy several opposing requirements.

The required external torque must be readily attainable. If $M_{t}$ is the maximum torque that can be applied and $\tau_{a}$ the shearing stress at the inner edge $r=a$ of the annulus, we have from (1)

$$
a=\sqrt{\frac{M_{t}}{2 \pi \tau_{a} h}} .
$$

The torque must be applied uniformly to the edges of the specimen. This makes it inadvisable to use clamps consisting of heavy clamping rings held together by individual bolts. In such a clamping arrangement one would expect higher shearing stresses to be transmitted near the bolts, where the clamping pressures are high, than at locations relatively far from the bolts. It would be better to cut a circular disk with a radius well in excess of $b$ from the material and then apply the torque to one or both faces of this disk through adhesive layers, as indicated in figure 2. The torque $M_{t}$ is then limited by the shear stress $\tau_{s}$ transmitted through the adhesive at the inner edge $r=a$ of the annulus.

The magnitude of $\tau_{s}$ can be computed from the torque $M_{t}$ on the assumption that each adhesive layer behaves like an elastic lamination of constant thickness connecting two rigid bodies that are rotated relative to each other. The shearing stress is then proportional to the distance $r$ from the axis of twist. If there is only one layer, the torque is equal to

$$
M_{t}=\int_{0}^{a} r 2 \pi r \tau d r=2 \pi \int_{0}^{a} r^{2} \tau_{s} \frac{r}{a} d r=\frac{\pi}{2} a^{3} \tau_{s}
$$

The torque $M_{t}$ produces a shearing stress $\tau_{a}$ at the inner radius of the annulus given by (1)

$$
\tau_{a}=\frac{M_{t}}{2 \pi a^{2} h} .
$$

With (16) this leads to the condition

$$
2 \pi a^{2} h \tau_{a} \leq \frac{\pi}{2} a^{3} \tau_{s}
$$

or

$$
a / h \geq 4 \tau_{a} / \tau_{s}
$$

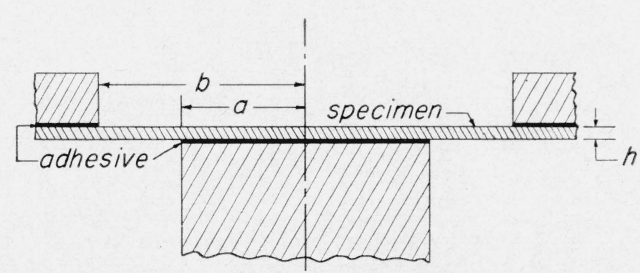

Figure 2. Cross-sectional view of annulus and grips. 
The sheet in the annulus must be stable against buckling into a pattern of shear wrinkles up to the highest torque applied. The authors have not found a solution for the buckling stress of an annulus. However, an approximation to this buckling stress is obtained from Southwell and Skan's solution for the infinitely long elastic strip with clamped edges [4]; with $\nu=0.3$ this reduces to

$$
\tau_{c r}=21.1 \frac{G h^{2}}{(b-a)_{2}} .
$$

The modulus of elasticity in shear, $G$, must be replaced by a reduced modulus $\bar{G}$, where $\bar{G}$ will be somewhere between $G$ and the tangent modulus at $\tau_{c r}$, since buckling must not take place until the material at $r=r_{1}$ has been stressed well into the plastic range. This leads to the condition

$$
\bar{\tau}<21.1 \frac{\bar{G} h^{2}}{(b-a)^{2}}
$$

where $\bar{\tau}$ denotes the shearing stress at the mean radius $c=(a+b) / 2$, of the annulus. Condition (21) sets an upper limit to the width $(b-a)$.

A lower limit is set by the condition that $(b-a)$ must be sufficient to accomodate the device for measuring relative displacement.

Another condition that must be satisfied is that the tensile strain in a radial direction is negligible. From figure 3 we see that this radial strain is in first approximation

$$
\epsilon_{r}=\frac{\overline{A^{\prime} B^{\prime}}-\overline{A B}}{\overline{A B}}=\frac{\overline{A^{\prime} B^{\prime}}}{\overline{A B}}-1=\frac{\sqrt{(b-a)^{2}+\left(v_{b}-v_{a}\right)^{2}}}{(b-a)}-1
$$

or with

$$
\begin{gathered}
\frac{v_{b}-v_{a}}{b-a}=\bar{\gamma} \\
\epsilon_{\tau}=\sqrt{1+\bar{\gamma}^{2}-1}=\frac{\bar{\gamma}^{2}}{2}-\cdots
\end{gathered}
$$

This must be small compared to the normal strain of the order $\bar{\gamma} / 2$ along lines at $45^{\circ}$ to the radial

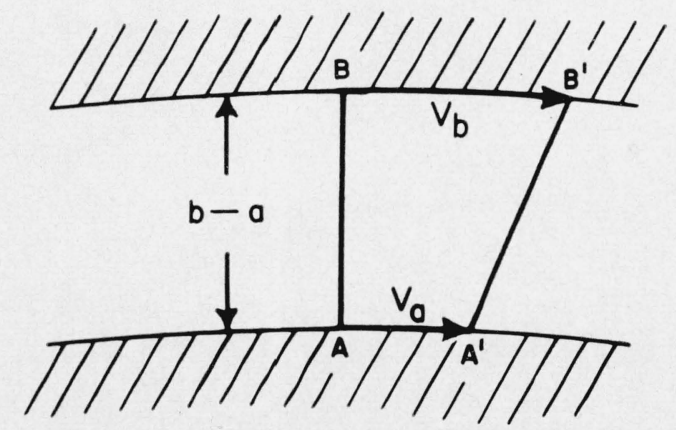

Figure 3. Diagramatic sketch for estimating radial strain due to relative circumferential displacement of inner and outer edges of the annulus. direction, that is,

From (24)

$$
\frac{\epsilon_{r}}{\bar{\gamma} / 2} \ll 1
$$

$$
\underset{\bar{\gamma} / 2}{\epsilon_{r}}=\bar{\gamma}-\ldots
$$

which is small compared to one in the range in which we are interested.

Let us apply these equations to the specific case of high-strength aluminum-alloy sheet with a vield strength in shear, $\tau_{1}$, of the order of $45,000 \mathrm{lb} / \mathrm{in}^{2}$ (tensile yield strength about $78,000 \mathrm{lb} / \mathrm{in}^{2}$ ) to be tested in a torsion machine with a capacity of 27,000 lb-in. Let us assume that a bonding material with a shear strength $\tau_{s}=4,500 \mathrm{lb} / \mathrm{in}^{2}{ }^{2}$ can be used for applying the torque to the specimen, and that a displacement measuring device with a width of $1 \mathrm{in}$. can be constructed to measure $\delta$, so that $b-a \geq 1$ in. Let us assume also that the stress at the inner edge exceeds the yield strength in shear by 20 percent $\tau_{a}=1.2 \tau_{1}=54,000 \mathrm{lb} / \mathrm{in}^{2}$ Substituting these quantities in (17) and (19) gives $a \leq 0.282 / \sqrt{h}$ and $a \geq 48 h$, respectively, or, $48 h \leq a \leq 0.282 / \sqrt{h}$. We obtain an upper limit for the thickness $h, h \leq 0.0326$ in., by going to the case in which $a$ is equal to its lower and its upper limit. The corresponding radius of the inner clamp is $a=0.282 / \sqrt{0.0326}=1.56 \mathrm{in}$. The outer and the mean radius become $b=a+1=$ $2.56 \mathrm{in} ; \quad c=2.06 \mathrm{in}$. The shearing stress at the mean radius is from (1) $\bar{\tau}=\tau_{a}\left(a^{2} / c^{2}\right)=31,000 \mathrm{lb} / \mathrm{in}^{2}{ }^{2}$

This calculation indicates that the annulus must have a thickness of less than 0.0326 in. for highstrength aluminum-alloy sheet with a yield strength in shear of the order of $45,000 \mathrm{lb} / \mathrm{in}^{2}{ }^{2}$

A lower limit for the thickness may be obtained by solving (21) for $h$. Since much of the material in the annulus will be in the elastic range, it seems reasonable to assume $3,000,000 \mathrm{lb} / \mathrm{in}^{2}$ (about threefourths the modulus of elasticity in shear) as an average value of $\bar{G}$. Then from (21)

$$
h>(b-a) \sqrt{\bar{\tau} / 21.1 \bar{G}}=0.0221 \mathrm{in} .
$$

We conclude that the thickness should be between 0.022 and $0.033 \mathrm{in}$. for this alloy tested under the assumed conditions.

\subsection{Method of Applying Torque}

Advice regarding the bonding medium for transmitting torque to the specimen was obtained from experts in the field, and it was decided to try cements of the Epoxy type upon recommendation of N. J. DeLollis of the National Bureau of Standards and D. M. Alstadt of the Lord Manufacturing Co., Erie, $\mathrm{Pa}$.

The shear strength of the cement was determined from torsion tests of specimens made by joining in a butt joint two pieces of aluminum-alloy rod 1.5 in. in diameter. Similar specimens were made from aluminum-alloy tubing $1.5 \mathrm{in}$. in diameter and 0.094 in. thick. The surfaces were cleaned with a degreasing solvent and Deoxidine. The bonding 
medium was EPON adhesive VI with 6 parts by weight of accelerator A to 100 parts of the adhesive. As soon as the adhesive was applied to one piece, the two pieces were pressed together until the thickness of adhesive was from 0.005 to 0.01 in. The pieces were held in position by clamps while the adhesive was cured for $45 \mathrm{~min}$. at from $200^{\circ}$ to $220^{\circ}$ F. The excess adhesive was removed from the outer surface of each specimen.

The maximum shear strength $\tau_{s}$, computed from these tests by substituting the torque at failure in (16) for the rod and in an analogous equation for the tube, was between 6,200 and $6,800 \mathrm{lb} / \mathrm{in}^{2}$ for the tubular specimens and between 6,000 and 6,700 $\mathrm{lb} / \mathrm{in}^{2}{ }^{2}$ for the cylindrical specimens.

Attempts to develop shear strength of this order in transmitting torque to a specimen of 0.032 -in. aluminum-alloy sheet bonded to a 3 -in. aluminumalloy cylinder were unsuccessful. This may have been due in part to stress concentration in the adhesive at the inner edge of the annulus. This stress concentration was relieved somewhat by machining a circumferential notch in the cylinder $0.01 \mathrm{in}$. from the bond and by bonding disks ( $\mathrm{A}$ and B, fig. 4) of 0.020 -in. aluminum-alloy sheet to the specimen.

The radii of $\mathrm{A}$ and $\mathrm{B}$ were 0.06 and 0.03 in., respectively, greater than the radius of cylinder C. The cylinder, the disks, the specimen $\mathrm{S}$, and the ring $\mathrm{R}$ were held coaxially by the pin $\mathrm{P}$ and the jig $J$ while the adhesive was being pressed out to a thickness of about 0.01 in. and during the curing of the adhesive. The jig was also used for locating the bolt holes in the ring and for holding the ring while it was being machined. The bond between the cylinder and disk B did not fail until the value of $\tau_{s}$ reached $4,670 \mathrm{lb} /$ in. $^{2}$ No difficulty was experienced with the bond between the specimen, which had a diameter of 6.5 in., and ring $R$, the inside diameter of which was 5 in.

Two torsion-testing machines were available for the tests, a Riehle testing machine of $60,000-1 b-i n$. capacity and an Amsler testing machine of $150-\mathrm{kg}-\mathrm{m}$ (13,000-lb-in.) capacity. In the Riehle machine the torque was transmitted to the weighing beam through a series of knife edges and linkages, which provided a very flexible mounting for one head of the machine. The specimen was first set up in this machine because of its greater capacity but was moved to the Amsler machine when it was found that good aline-

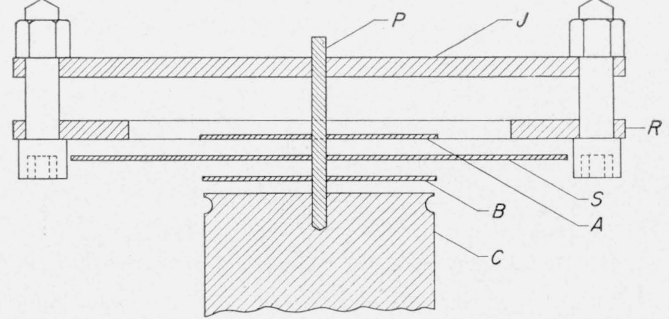

Figure 4. Expanded cross-sectional view of specimen S, disks $A$ and $B$, cylinder $C$ and ring $R$, positioned by jig $J$, and centered with pin $P$ for bonding.

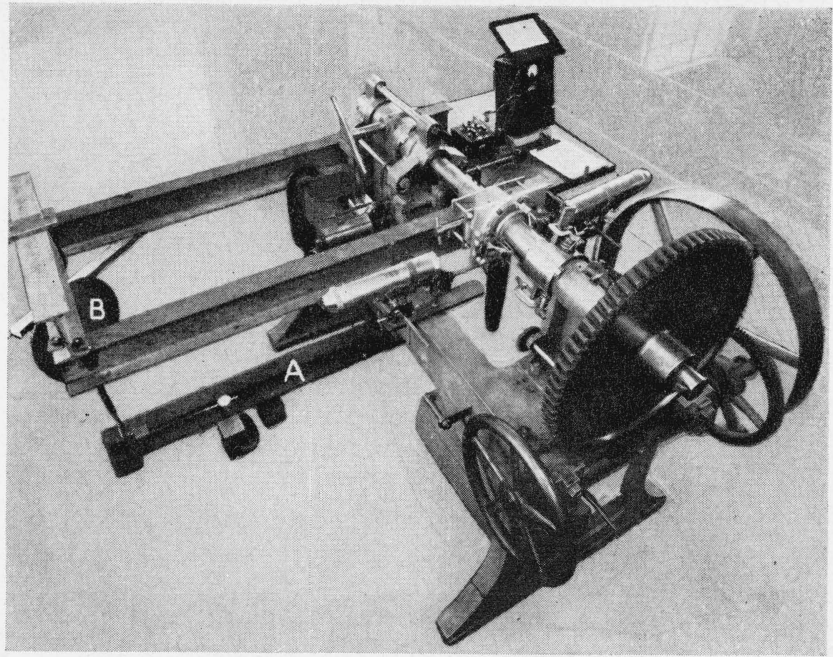

Figure 5. Modified Amsler torsion machine.

ment could not be maintained. The alinement of the weighing head of the Amsler machine is maintained by ball bearings. The 13,000-lb-in. capacity, measured by rotation of the pendulum, was insufficient for the specimen. To increase the capacity, the weighing system was changed to the simple lever and scale type, utilizing the pendulum only for attaching the arm A, figure 5. The effective length of the lever was 48 in., and the scale was the spring balance B, having a capacity of $600 \mathrm{lb}$. During the test the pendulum was maintained in the zero position so that its moment would not affect the torque measurement. The specimen assembly was mounted in the testing machine by bolting the ring to the plate $H$, figure 6 , attached to the dynamometer head of the machine. The holes in the plate had been located by the jig $\mathrm{J}$, shown in figure 4. The cylinder was centered in the loading head of the machine.

\subsection{Method of Measuring Deformation}

The device for measuring deformation of the specimen is shown in position in figure 7 . The

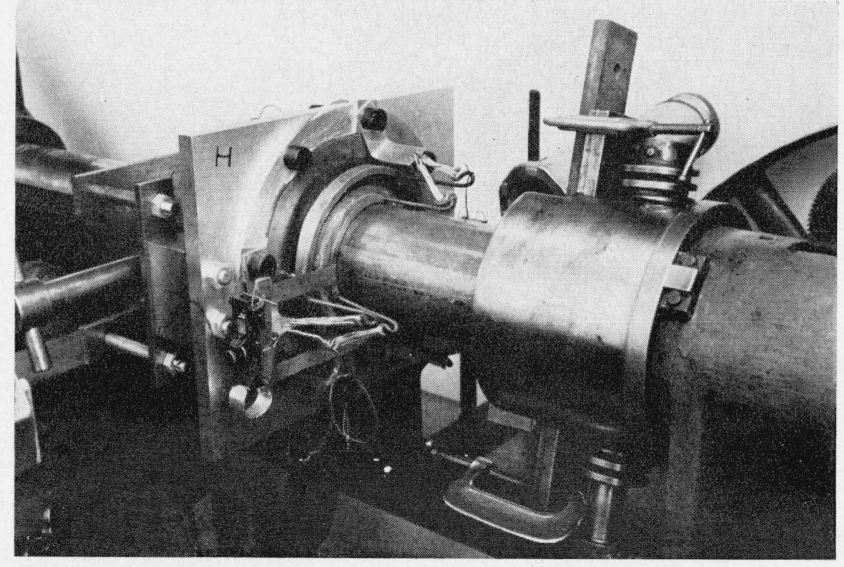

FIgURE 6. Specimen ready for test in modified Amsler torsion machine. 


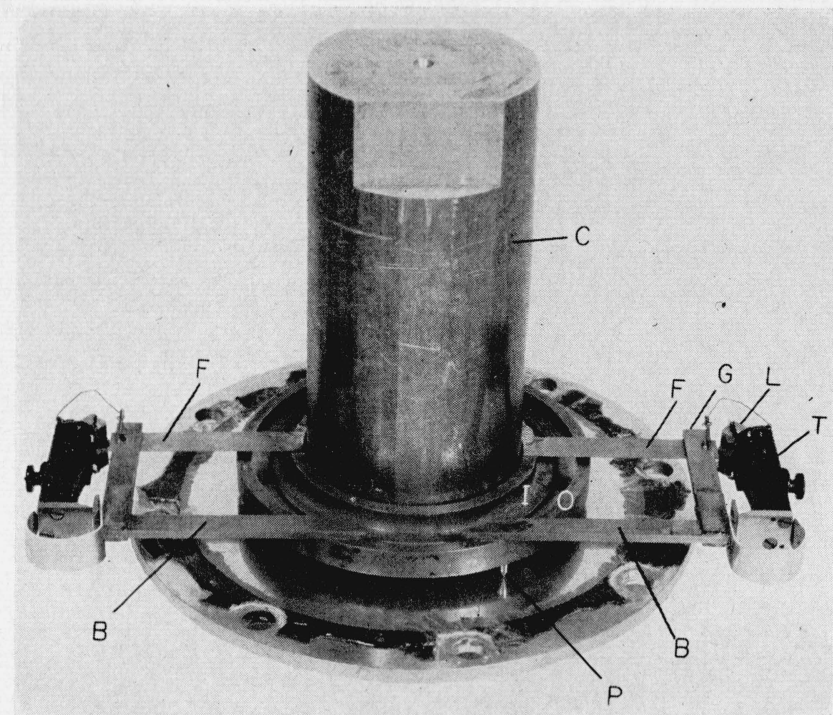

FiguRE 7. Gage for measuring relative circumferential displacement.

purpose of the device is to measure the relative rotation between two circles concentric with the axis of the cylinder $\mathrm{C}$ having radii $r_{2}$ and $r_{1}$ of 2.375 and 1.625 in., respectively. These circles are defined by conical points, such as $\mathrm{P}, 120^{\circ}$ apart, mounted on the rings $\mathrm{O}$ and $\mathrm{I}$, respectively. The relative rotation is measured by measuring the relative tangential motion between the two rings. To increase the sensitivity and reduce any errors due to bending of the specimen, the motion between the ends of the arms B and $\mathrm{F}$ is measured. Due account is taken of the radius at which the motion is measured. Each arm B is attached rigidly to the outer ring $\mathrm{O}$ and provides physical support to the body of a Tuckerman optical strain gage T. Each arm F, attached to the inner ring $\mathrm{I}$, is flexible in the direction of the axis of the specimen and rigid in the direction of rotation. The rotating lozenge $\mathrm{L}$ of each gage is held against the outer end of an arm F by a pair of hairpin springs, one attached to each end of a rod through a hole in $\mathrm{F}$ and to a knob on each side of the gage. Guides $G$ were rigidly attached to each arm B to limit the motion of the end of arm $\mathrm{F}$ to a plane perpendicular to the axis of the gage lozenge. The circumferential displacement $\delta$ of the circle with radius $r_{1}$ with respect to the circle with radius $r_{2}$ is given by

$$
\delta=e \frac{r_{2}}{r_{L}}
$$

where $e$ is the average displacement measured by the gages, and $r_{L}$ is the average of the radii to the points of contact of gage lozenges with the arms $\mathrm{F}$.

\section{Results}

A disk of 0.032 -in. $75 \mathrm{~S}-\mathrm{T} 6$ aluminum-alloy sheet was prepared and tested in shear as described above.

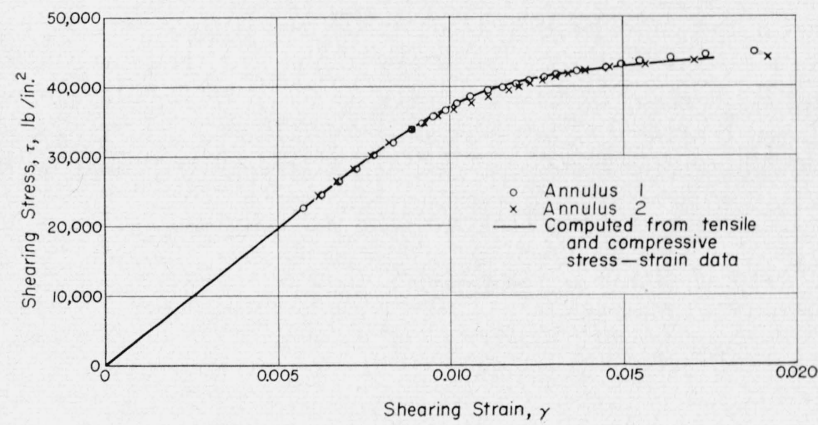

Figure 8. Stress-strain curve in shear for a sheet of 75S-T6 aluminum alloy 0.032 in. thick.

The test portion of the disk consisted of an annulus with inner and outer radii of 1.56 and 2.5 in., respectively. The test was carried to a shearing stress of $48,000 \mathrm{lb} / \mathrm{in}^{2}{ }^{2}$ and a shearing strain of more than 0.05 at the inner gage ring before the adhesive at the inner edge of the annulus broke at a shear stress in the adhesive, as computed from (16), of 4,670 $\mathrm{lb} /$ in. $^{2}$. A second annulus reached a shearing stress of over $45,000 \mathrm{lb} / \mathrm{in}^{2}$. The data for both annuli up to a strain of about 0.019 are plotted in figure 8, together with a stress-strain curve in shear computed from an average stress-strain curve obtained from tests of tensile and compressive specimens from the same sheet. This curve was computed, in accordance with octahedral shear theory [5] from

$$
\tau=\sigma / \sqrt{3} \quad \text { and } \gamma=\sqrt{3}\left(\epsilon-\frac{17}{150} \frac{\sigma}{E}\right)
$$

where $\sigma$ is the average of the stresses corresponding to the strain $\epsilon$ obtained in tensile and compression tests of specimens in the direction of rolling and across the direction of rolling of the sheet, and $E$ is the average of the moduli of elasticity in tension and compression. The shear data are in good agreement with the derived curve. The modulus of elasticity in shear for each specimen differed by less than 1 percent from that computed from $E$ and the nominal value of Poisson's ratio (0.33) for this alloy.

\section{Conclusions}

The twisting test of an annulus appears feasible for determining the stress-strain relation in shear of isotropic sheet metal in thicknesses below those which can be tested by twisting a square plate of the material. Special precautions must be taken to obtain a good bond for transmitting the shearing stresses to the specimen and to have good alinement between the two heads of the torsion-testing machine.

Using this method, the stress-strain curve in shear was determined for 0.032 -in. aluminum alloy $75 \mathrm{~S}-\mathrm{T} 6$ sheet up to a strain of 0.05 , which is far beyond the elastic range. The stress-strain curve thus obtained was in good agreement with one derived from the stress-strain curve of the same material in tension and compression, on the assumption that the octahedral shear stress in the metal was a function of the octahedral shear strain only. 
The authors acknowledge the assistance of members of the Engineering Mechanics Section, in particular that of A. E. McPherson, D. F. Hoeschele, Jr., H. L. Byers, Jr., and R. H. Harwell, Jr.

\section{References}

[1] Walter Ramberg and James A. Miller, Twisted square plate method and other methods for determining the shear stress-strain relation of flat sheet, J. Research NBS 50, (1953) RP2397.
[2] S. Timoshenko and J. N. Goodier, Theory of elasticity (McGraw-Hill Book Co., Inc., New York, N. Y., 1951).

[3] Philipp Frank and Richard von Mises, Die Differentialund Integralgleichungen der Mechanik und Physik (Vieweg, Braunschweig, 1930).

[4] R. B. Southwell and S. W. Skan, On the stability under shearing forces of a flat elastic strip, Proc. Roy. Soc. London 105, 582-607 (1924).

[5] A. Nadai, Theory of flow and fracture of solids, 1, 103, 115 (McGraw-Hill Book Co., Inc., New York, N. Y., 1950).

Washington, July 17, 1952. 